\title{
Do no harm: a paradigm shift in the unchecked use of antidepressants
}

\author{
Debbi Ann Morrissette*
}

Neuroscience Education Institute, Carlsbad, California, USA

First published online 9 February 2017

This work is dedicated to S., the actual patient described herein.

The following true case scenario is one that the new paradigm shift in recognizing and treating mixed features of major depressive episodes is poised to prevent. Asking about subsyndromal symptoms of mania/hypomania, as well as asking about family history, will potentially alter clinical practice and change outcomes for many lives.

The Case. A 17-year-old male presents to his primary care provider with symptoms of depression (anhedonia, feelings of worthlessness, etc.), and he is placed on antidepressant monotherapy and sent on his way. Approximately 2 months later, the patient experiences a full-blown manic episode with suicidal ideation. He is hospitalized, and a more appropriate treatment (in this case, an antipsychotic with mood-stabilizing properties) is initiated. However, this 17-year-old patient has already lost his job, his long-term girlfriend, and his chance at entering college with his peer group, all as a result of his manic episode. As it turns out, the mother of the patient had been diagnosed with bipolar disorder I, and the patient himself had at times exhibited subsyndromal symptoms of hypomania (including irritability, distractibility, and racing thoughts). His first-line clinician had never asked about family history or assessed for anything beyond symptoms of unipolar depression, and the patient did not know the importance of mentioning these things. The solution could have been a short conversation about family history and a few minutes spent filling out a simple questionnaire while in the waiting room to possibly avoid this devastating outcome-a young life seemingly damaged right as it was beginning.

* Address for correspondence: Debbi Ann Morrissette, Senior Medical Writer, Neuroscience Education Institute, 5900 La Place Court, Suite 120, Carlsbad, California 92008, USA.

(Email: dmorrissette@neiglobal.com)
With our newly published guidelines for diagnosis and treatment of mood disorders all along the spectrum, ${ }^{1}$ we (myself along with the top mood-disorder experts from around the world) are advocating that every patient who presents with symptoms of depression be thoroughly screened for family history of bipolar disorders and symptoms of (hypo)mania. I would argue that our guidelines not be viewed as something to instruct the diagnosis and treatment of some "obscure" mixed depression (rarely encountered and only for the most advanced psychiatric professionals to deal with), but as guidelines for what to do whenever a patient with seemingly "unipolar" depression comes to us for help.

These newly developed guidelines do not necessarily suggest that an antidepressant should never be used-for many (approximately half) of the patients with symptoms of depression, the antidepressant class of medications is relatively effective and safe. What we argue is that antidepressant monotherapy be earned-meaning that, before any patient is placed on an antidepressant, we have done all in our power to ensure that any symptoms of (hypo)mania and positive family history have been aggressively ruled out. And in cases where antidepressant monotherapy is initiated, continuous and frequent monitoring for the emergence of (hypo)manic symptoms is our obligation to the patients (and their families) who are trusting us with their care.

The mixed depression guidelines may be especially critical when it comes to the pediatric population. One indication that depression may actually NOT be unipolar in nature is young age of onset. When any patient under the age of 18 presents with symptoms of depression, mixed features or bipolarity should actually be expected and screened for thoroughly, intensely, and with no stone left unturned. If evidence that symptoms fall outside the realm of unipolar are suspected, antidepressant monotherapy should probably not be the treatment of choice. Not only may antidepressant monotherapy spark a switch 
to mania and possible emergence of suicidality, but the evidence indicates that, with the exception of fluoxetine, antidepressants DON'T WORK when it comes to pediatric depression. The potential risks therefore far outweigh any potential benefits. As frightening as it may seem to place such young patients on such "dangerous" agents as atypical antipsychotics (with potential side effects, such as weight gain or movement disorders) or mood stabilizers (with inconvenient monitoring requirements) rather than the "safe" antidepressant options (with potential risks of mania, hospitalization, and suicide), it seems a choice that we must become more comfortable with. Suicide is arguably the worst possible outcome-the one any clinician dreads most for any patient under his or her care. The prescribing of an antidepressant should therefore never be taken lightly or as an always safe course of action.

We have the power to prevent such catastrophic outcomes as suicidality, manic/hypomanic episodes, or even simply failure to respond. However, it is going to require a paradigm shift in the current thinking that antidepressants are always the first choice for anyone with any depressive symptom and always safe options compared to antipsychotics or mood-stabilizing agents. Antidepressant monotherapy is possibly not the best choice for many (half?) of all patients with symptoms of depression-suicidality is not an acceptable treatmentinduced side-effect risk.

\section{Disclosures}

Dr. Morrissette hereby declares that she has no conflicts of interest to disclose.

\section{REFERENCE:}

1. Stahl SM, Morrissette DA, Faedda G, et al. Guidelines for the recognition and management of mixed depression. CNS Spectr. 2017; 22(2): 203-219. 